\title{
Using Geminal Dicationic Ionic Liquids as Solvents for High Temperature Organic Reactions
}

\author{
Supporting Information \\ Xinxin Han, and Daniel W. Armstrong* \\ Chemistry Department, Iowa State University, Ames, IA, 50011
}

Material preparations

2

Spectra of compounds

4 


\section{Genaral Methods}

NMR spectra were recorded using a Varian $300 \mathrm{MHz}$ NMR and samples were prepared in deuterated solvents. The HPLC system included two Shimadzu LC-10AD pumps, one Shimadzu SPD-6A UV VWD detector, and one Shimadzu CR 601 integrator. The C18 column is obtained from Advanced Separation Technologies (Whippany, NJ, USA). The UV detection wavelength for all the compounds is $254 \mathrm{~nm}$ and the flow rate of mobile phase is $1.0 \mathrm{~mL} / \mathrm{min}$. The mobile phase is the mixture of acetonitrile and water. All chemicals were purchased form Aldrich and used directly unless otherwise noted. All the three dicationic ionic liquids were prepared following the previous procedure. ${ }^{1}$ The synthesis of $m$-substituted allyl phenyl ether is also illustrated elsewhere ${ }^{2}$.

Isomerization of carvone to carvacrol (1). $300 \mathrm{mg}$ carvone was dissolved in $5 \mathrm{~mL}$ dicationic ionic liquid and the mixture was heated to $250{ }^{\circ} \mathrm{C}$ or $300{ }^{\circ} \mathrm{C}$ under argon. The reaction was monitored by TLC. After the reaction was completed, ethyl ether $(5 \times 5 \mathrm{~mL})$ was used to extract the product from the cooled down ionic liquid solution. Ethyl ether was removed under vacume and the residue was purified by flash chromatography (hexanes/EtOAc). Yield was different according to different reaction condition. ${ }^{1} \mathrm{H}$ NMR $\left(300 \mathrm{MHz}, \mathrm{CDCl}_{3}\right) \delta 7.04(\mathrm{~d}, J=7.8 \mathrm{~Hz}, 1 \mathrm{H}), 6.73\left(\mathrm{dd}, J_{l}=7.8 \mathrm{~Hz}, J_{2}=1.5 \mathrm{~Hz}\right.$, $1 \mathrm{H}), 6.66(\mathrm{~d}, J=1.6 \mathrm{~Hz}, 1 \mathrm{H}), 4.58(\mathrm{br}, 1 \mathrm{H}), 2.83(\mathrm{~m}, J=6.9 \mathrm{~Hz}, 1 \mathrm{H}), 2.22(\mathrm{~s}, 3 \mathrm{H}), 1.22(\mathrm{~d}, J=6.9 \mathrm{~Hz}$, $6 \mathrm{H}) .{ }^{13} \mathrm{C} \mathrm{NMR}\left(75 \mathrm{MHz}, \mathrm{CDCl}_{3}\right) \delta 153.8,148.7,131.1,121.1,119.0,113.3,33.9,24.2,15.6$. The NMR results are matched with literature ${ }^{3}$.

Claisen Rearrangement (2-5). $200 \mathrm{mg}$ allyl phenyl ether was dissolved in $5 \mathrm{~mL}$ dicationic ionic liquid and the mixture was heated to $250{ }^{\circ} \mathrm{C}$ or $300{ }^{\circ} \mathrm{C}$ under argon for 10 minutes (by TLC). After the reaction was completed, ethyl ether $(5 \times 5 \mathrm{~mL})$ was used to extract the product from the cooled down ionic liquid solution. Ethyl ether was removed under vacume and the residue was purified by flash chromatography (hexanes/EtOAc). Yield was different according to different reaction condition. Similar procedure was used for the rearrangement of $m$-substituted allyl phenyl ether.

2-allylphenol: ${ }^{1} \mathrm{H}$ NMR $\left(300 \mathrm{MHz}, \mathrm{CDCl}_{3}\right) \delta$ 7.18-7.13 (m, 2H), 6.94-6.89 (m, 1H), $6.83(\mathrm{~d}, J=8.1 \mathrm{~Hz}$, $1 \mathrm{H}), 6.09-5.98(\mathrm{~m}, 1 \mathrm{H}), 5.22-5.19(\mathrm{~m}, 1 \mathrm{H}), 5.16-5.15(\mathrm{~m}, 1 \mathrm{H}), 5.08(\mathrm{~s}, 1 \mathrm{H}), 3.44(\mathrm{~d}, J=6.3 \mathrm{~Hz}, 2 \mathrm{H})$.

${ }^{13} \mathrm{C}$ NMR $\left(75 \mathrm{MHz}, \mathrm{CDCl}_{3}\right) \delta 154.3,136.7,130.7,128.1,125.6,121.2,116.7,116.0,35.3$. The NMR results are matched with literature ${ }^{4}$.

2-Allyl-5-methoxyphenol. ${ }^{1} \mathrm{H}$ NMR $\left(300 \mathrm{MHz}, \mathrm{CDCl}_{3}\right) \delta 6.99(\mathrm{~d}, J=8.1 \mathrm{~Hz}, 1 \mathrm{H}), 6.46\left(\mathrm{dd}, J_{l}=8.1\right.$ $\left.\mathrm{Hz}, J_{2}=2.4 \mathrm{~Hz}, 1 \mathrm{H}\right), 6.42(\mathrm{~d}, J=2.4 \mathrm{~Hz}, 1 \mathrm{H}), 6.07-5.94(\mathrm{~m}, 1 \mathrm{H}), 5.20-5.16(\mathrm{~m}, 1 \mathrm{H}), 5.16-5.12(\mathrm{~m}, 1 \mathrm{H})$, $5.10(\mathrm{~s}, 1 \mathrm{H}), 3.77(\mathrm{~s}, 3 \mathrm{H}), 3.35(\mathrm{~d}, J=6.3 \mathrm{~Hz}, 2 \mathrm{H}) .{ }^{13} \mathrm{C}$ NMR $\left(75 \mathrm{MHz}, \mathrm{CDCl}_{3}\right)$ $\delta 159.8,155.3,137.0,131.1,117.5,116.5,106.6,102.3,55.6,34.8$. The NMR results are matched with literature ${ }^{5}$.

2-Allyl-3-methoxyphenol. ${ }^{1} \mathrm{H}$ NMR $\left(300 \mathrm{MHz}, \mathrm{CDCl}_{3}\right) \delta 7.08(\mathrm{t}, J=8.1 \mathrm{~Hz}, 1 \mathrm{H}), 6.50(\mathrm{~d}, J=8.1 \mathrm{~Hz}$, 
$1 \mathrm{H}), 6.49(\mathrm{~d}, J=8.1 \mathrm{~Hz}, 1 \mathrm{H}), 6.05-5.92(\mathrm{~m}, 1 \mathrm{H}), 5.15-5.13(\mathrm{~m}, 1 \mathrm{H}), 5.11-5.06(\mathrm{~m}, 1 \mathrm{H}), 5.01(\mathrm{~s}, 1 \mathrm{H})$, $3.81(\mathrm{~s}, 3 \mathrm{H}), 3.47\left(\mathrm{dt}, J_{l}=6.0 \mathrm{~Hz}, J_{2}=1.8 \mathrm{~Hz}, 2 \mathrm{H}\right) .{ }^{13} \mathrm{C} \mathrm{NMR}\left(75 \mathrm{MHz}, \mathrm{CDCl}_{3}\right)$ $\delta 158.4,155.4,136.5,127.8,115.7,113.8,109.0,103.6,56.0,27.6$. The NMR results are matched with literature ${ }^{5}$.

2-Allyl-5-trifluoromethylphenol. ${ }^{1} \mathrm{H}$ NMR $\left(300 \mathrm{MHz}, \mathrm{CDCl}_{3}\right) \delta 7.22(\mathrm{~d}, J=8.1 \mathrm{~Hz}, 1 \mathrm{H}), 7.15(\mathrm{~d}, J=$ $8.1 \mathrm{~Hz}, 1 \mathrm{H}), 7.07(\mathrm{~s}, 1 \mathrm{H}), 6.07-5.94(\mathrm{~m}, 1 \mathrm{H}), 5.23-5.21(\mathrm{~m}, 1 \mathrm{H}), 5.19(\mathrm{~s}, 1 \mathrm{H}), 5.16-5.15(\mathrm{~m}, 1 \mathrm{H}), 3.46$ $(\mathrm{d}, J=6.3 \mathrm{~Hz}, 2 \mathrm{H}) .{ }^{13} \mathrm{C} \mathrm{NMR}\left(75 \mathrm{MHz}, \mathrm{CDCl}_{3}\right) \delta 154.4,135.5,131.1,130.5(\mathrm{q}, J=32.4$ $\mathrm{Hz}), 129.6(\mathrm{q}, J=1.2 \mathrm{~Hz}), 124.1(\mathrm{q}, J=270.3 \mathrm{~Hz}), 117.9(\mathrm{q}, J=3.9 \mathrm{~Hz}), 117.6,113.0(\mathrm{q}, J=3.9$ $\mathrm{Hz}), 35.1$.

2-Allyl-3-trifluoromethylphenol. ${ }^{1} \mathrm{H}$ NMR $\left(300 \mathrm{MHz}, \mathrm{CDCl}_{3}\right) \delta$ 7.29-7.20 (m, 2H), 7.04-7.01 (m, $1 \mathrm{H}), 6.05-5.92(\mathrm{~m}, 1 \mathrm{H}), 5.24(\mathrm{~s}, 1 \mathrm{H}), 5.20-5.15(\mathrm{~m}, 1 \mathrm{H}), 5.12-5.10(\mathrm{~m}, 1 \mathrm{H}), 3.58(\mathrm{~d}, J=5.7 \mathrm{~Hz}, 2 \mathrm{H})$.

${ }^{13} \mathrm{C}$ NMR $\left(75 \mathrm{MHz}, \mathrm{CDCl}_{3}\right) \delta 155.6,135.4,130.3(\mathrm{q}, J=29.4 \mathrm{~Hz}), 127.9,124.5(\mathrm{q}, J=272.3$ $\mathrm{Hz}), 124.1(\mathrm{q}, J=1.6 \mathrm{~Hz}), 120.3(\mathrm{q}, J=1.0 \mathrm{~Hz}), 118.8(\mathrm{q}, J=5.8 \mathrm{~Hz}), 116.96,31.2(\mathrm{q}, J=2.1$ $\mathrm{Hz})$.

2-Allyl-5-methylphenol and 2-Allyl-3-methylphenol. These two compounds cannot be separated by flash chromatography. ${ }^{1} \mathrm{H}$ NMR $\left(300 \mathrm{MHz}, \mathrm{CDCl}_{3}\right)$ 7.05-6.98 (m, 2H), 6.79-6.65 (m, 4H), 6.08-5.91 $(\mathrm{m}, 2 \mathrm{H}), 5.18-4.99(\mathrm{~m}, 4 \mathrm{H}), 4.86(\mathrm{~s}, 1 \mathrm{H}), 4.79(\mathrm{~s}, 1 \mathrm{H}), 3.43\left(\mathrm{dt}, J_{l}=5.7 \mathrm{~Hz}, J_{2}=1.8 \mathrm{~Hz}, 2 \mathrm{H}\right), 3.38(\mathrm{~d}, J$ $=6.3 \mathrm{~Hz}, 2 \mathrm{H}), 2.29(\mathrm{~s}, 6 \mathrm{H})$. The ${ }^{1} \mathrm{H}$ NMR result is matched with literature ${ }^{5}$.

Diels-Alder reaction (6). $178 \mathrm{mg}$ anthracene and $172 \mathrm{mg}$ diethyl fumarate were dissolved in $5 \mathrm{~mL}$ dicationic ionic liquid and the mixture was heated to $220{ }^{\circ} \mathrm{C}$ under argon for 10 minutes. After the reaction was completed, ethyl ether $(5 \mathrm{x} 5 \mathrm{~mL})$ was used to extract the product from the cooled down ionic liquid solution. Ethyl ether was removed under vacume and the residue was purified by flash chromatography (hexanes/EtOAc). Yield was different according to different reaction condition.

${ }^{1} \mathrm{H}$ NMR (300 MHz, $\mathrm{CDCl}_{3}$ ) $\delta$ 7.35-7.32 (m, 2H), 7.24-7.21 (m, 2H), 7.14-7.08 (m, 4H), 4.73 (s, 2H), 4.13-4.00 (m, 4H), $3.42(\mathrm{~s}, 2 \mathrm{H}), 1.22(\mathrm{t}, J=7.2 \mathrm{~Hz}, 6 \mathrm{H}) .{ }^{13} \mathrm{C}$ NMR $\left(75 \mathrm{MHz}, \mathrm{CDCl}_{3}\right)$ $\delta 172.6,142.3,140.6,126.6,126.4,124.8,124.0,61.2,48.0,47.0,14.5$. The NMR results are matched with literature ${ }^{6}$.

(1) Anderson, J. L.; Ding, R.; Ellern, A.; Armstrong, D. W. J. Am. Chem. Soc. 2005, 127, 593-604.

(2) White, W. N.; Slater, C. D. J. Org. Chem. 1961, 26, 3631-3638.

(3) From website: http://www.sigmaaldrich.com/spectra/fnmr/FNMR011649.PDF

(4) From website: http://www.sigmaaldrich.com/spectra/fnmr/FNMR009224.PDF

(5) Gozzo, F. C.; Fernandes, S. A.; Rodrigues, D. C.; Eberlin, M. N.; Marsaioli, A. J. J. Org. Chem. 2003, 68, 5493-5499.

(6) Garrigues, B.; Laporte, C.; Laurent, R.; Laporterie, A.; Dubac, J. Liebigs Ann. 1996, 739-741. 


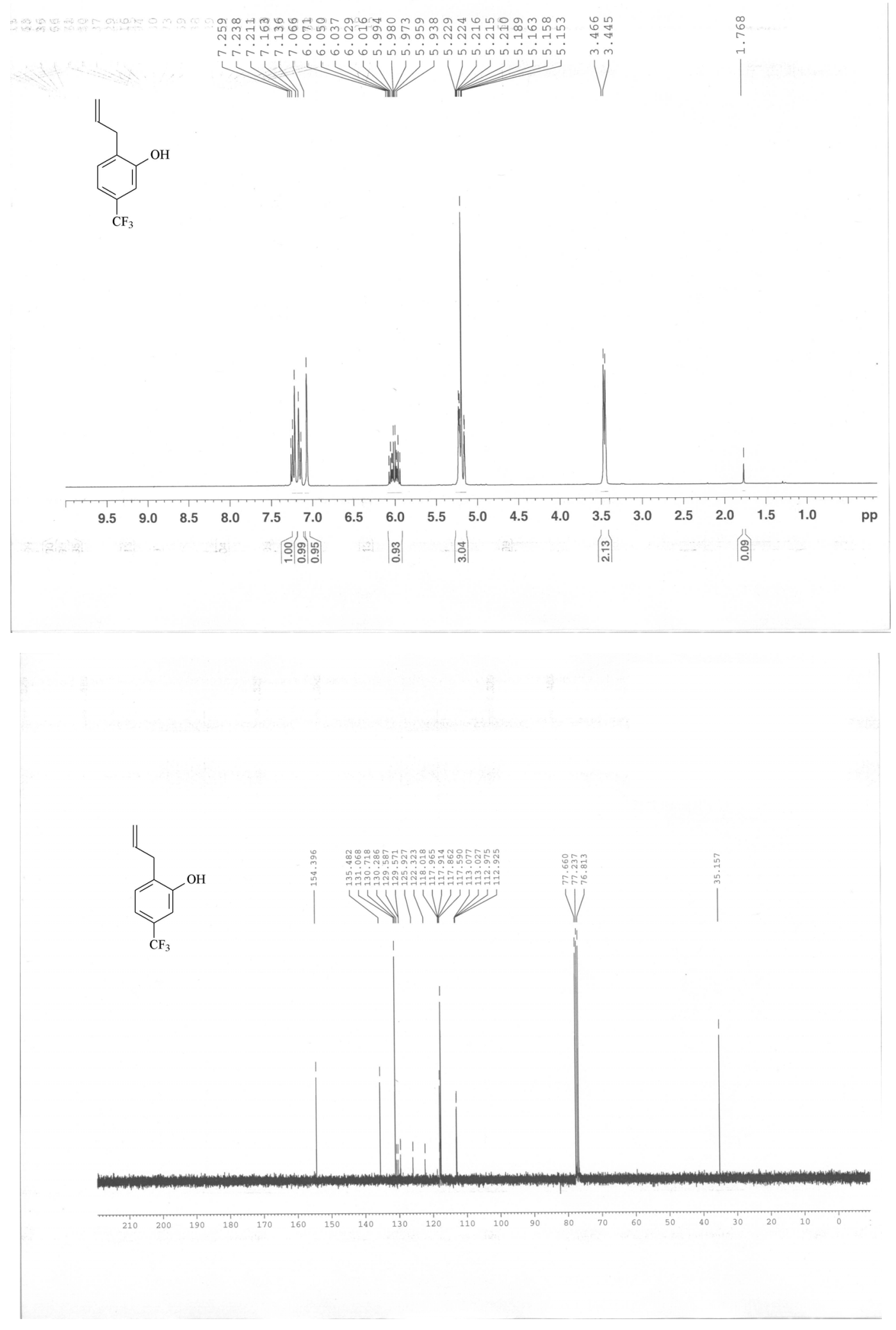




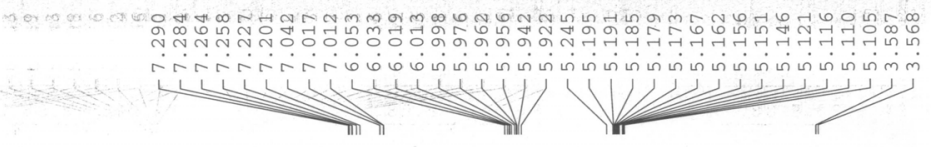

(NF
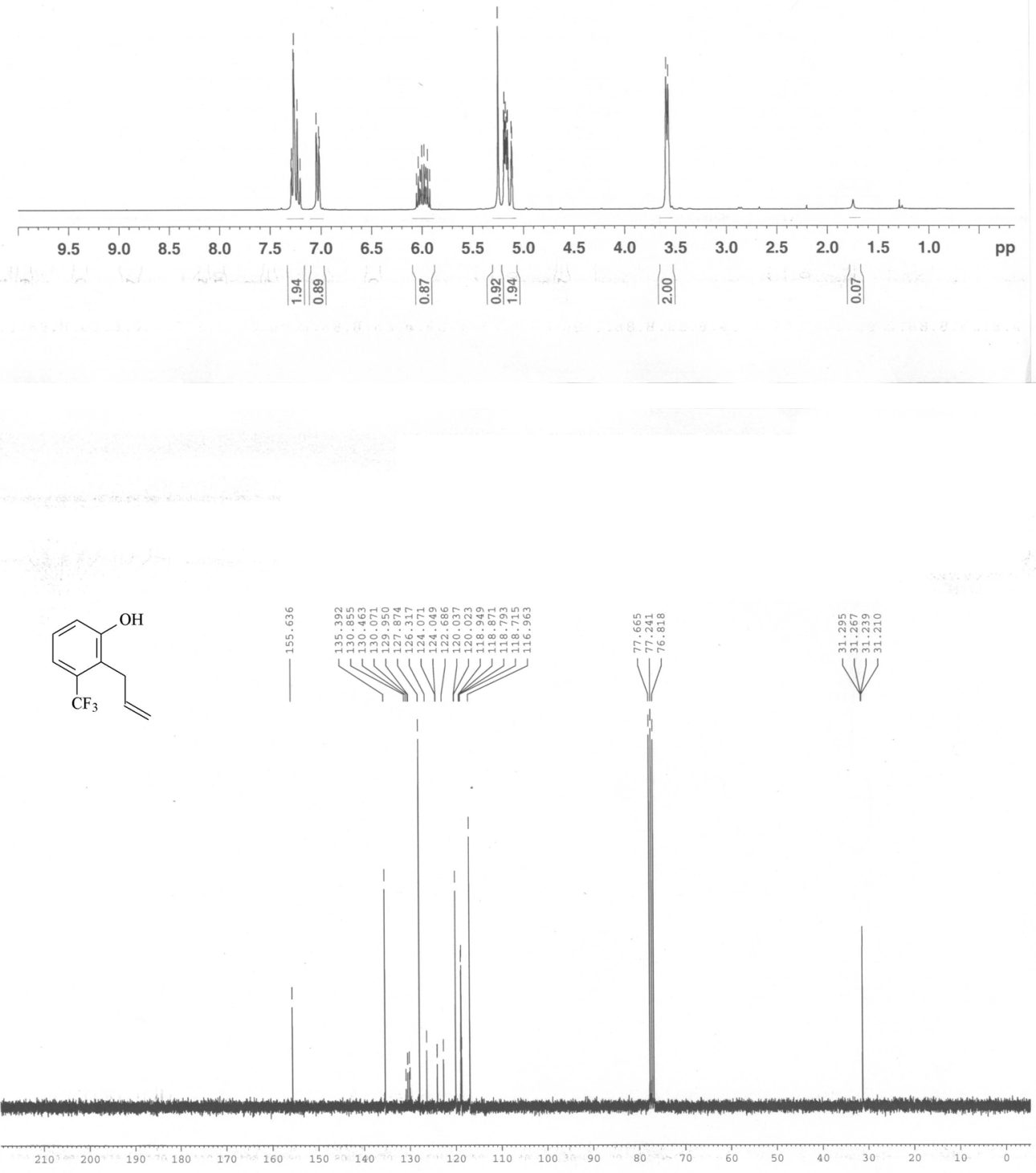